\title{
Eletrocatálise da reação de redução de oxigênio sobre eletrodos de grafite modificados com ftalocianina tetracarboxilada de ferro ${ }^{\S}$
}

\author{
J. A. P. Chaves ${ }^{1}$, M. F. A. Araújo ${ }^{2}$, J. de J. G. Varela Júnior ${ }^{2}$, A. A. Tanaka ${ }^{2 *}$ \\ ${ }^{1}$ Centro Universitário do Maranhão -CEP 65075-012 -São Luís - MA - Brasil \\ ${ }^{2}$ Departamento de Química - Universidade Federal do Maranhão - CEP 65.080-040 -São Luís - MA - Brasil
}

\begin{abstract}
Resumo: Este trabalho apresenta um estudo das propriedades eletroquímicas de eletrodos de grafite modificados, em níveis de monocamadas, com ftalocianina tetracarboxilada de ferro, FeTcPc, em soluções aquosas. Em meio alcalino, os eletrodos modificados apresentaram alta atividade eletrocatalítica para a reação de redução de oxigênio, comparável com a do eletrodo de platina. A reação processa-se de acordo com uma cinética de primeira ordem com relação ao oxigênio dissolvido, um mecanismo envolvendo 4 elétrons e associado com o processo redox $\mathrm{Fe}(3+) \mathrm{TcPc} / \mathrm{Fe}(2+) \mathrm{TcPc}$.
\end{abstract}

Palavras-chave: redução de oxigênio; metaloftalocianinas; eletrodos modificados.

\section{Introdução}

A eletroquímica do oxigênio tem despertado o interesse de muitos grupos de pesquisa devido a sua importância no desenvolvimento de sistemas eletroquímicos para conversão e armazenamento de energia, como as células a combustível, baterias metal-ar e eletrolisadores, bem como em sínteses químicas, processos biológicos e de corrosão. Assim, nas últimas décadas, particularmente motivado pelo desenvolvimento da tecnologia de células a combustível, muitos esforços têm sido dedicados ao estudo da reação de redução de oxigênio (RRO) em soluções aquosas ácidas e alcalinas sobre diferentes materiais de eletrodo [1-15]. Em eletrólitos ácidos, platina e ligas de platina ainda são consideradas as melhores opções para a redução de oxigênio, tanto em termos dos mais baixos sobrepotenciais desejáveis para promover a reação quanto da estabilidade requerida para operação como catodo em uma célula a combustível. Apesar dos esforços, os desafios da complexidade cinética e da necessidade de superação de uma barreira de energia de ativação significativa ainda permanecem e requerem o desenvolvimento de catalisadores alternativos eficientes e de baixo custo, associados com informações mais detalhadas dos mecanismos reacionais envolvidos.

Neste sentido, muitos estudos da atividade eletrocatalítica de eletrodos de carbono e grafite modificados com macrociclos de metais de transição, particularmente, metaloftalocianinas e metaloporfirinas, para a redução de oxigênio em soluções aquosas têm sido realizados [16-35]. Estes compostos pertencem a uma classe que fornecem oportunidades singulares para se examinar em detalhes os fatores envolvidos na ativação e

* e-mail tanaka@ufma.br

§ Dedicado ao Prof. Dr. Ernesto Rafael González, IQSC-USP, pelas suas contribuições ao desenvolvimento da Eletroquímica no Brasil. 
posterior redução da molécula de oxigênio. Tais fatores envolvem os efeitos associados com substituições nas posições axiais e periféricas do anel macrociclo, a natureza do centro metálico e o grau de conjugação do anel. Estudos da reatividade destes compostos com a molécula de oxigênio também são importantes para esclarecimentos de processos biológicos, de fotossíntese e de catálise. Adicionalmente, aspectos teóricos relacionados com este tema também despertam muita atenção, uma vez que permitem revelar o papel de efeitos localizado e coletivo, bem como estabelecer relações entre estrutura química e atividade catalítica.

Uma análise dos estudos das propriedades eletrocatalíticas dos eletrodos de carbono e grafite modificados com metaloftalocianinas e metaloporfirinas para a reação de redução de oxigênio em soluções aquosas [16-35] revelam que o metal de transição atua como o centro ativo e que a atividade eletrocatalítica depende principalmente das propriedades redox do eletrodo modificado, que muitas vezes não se apresentam explicitamente definidas e são inferidas de estudos realizados em solventes não aquosos. Além disso, dentre os diferentes metais que a princípio podem ser incorporados como centros metálicos, cobalto e ferro têm se destacados como os mais promissores para utilizações em catodos de oxigênio, por suas atividades eletrocatalíticas e estabilidade apresentadas nos experimentos, principalmente em soluções aquosas alcalinas. Enquanto a maioria dos complexos de cobalto catalisam a reação por meio de um processo envolvendo 2 elétrons e formação de peróxido de hidrogênio como principal produto (mecanismo peróxido), os de ferro são mais eficientes e promovem a redução através da transferência de 4 elétrons (mecanismo direto) e produção de água [2-4].

Entre os estudos da atividade eletrocatalítica de eletrodos de carbono e grafite modificados com metaloftalocianinas de ferro para a redução de oxigênio [16-35] destaca-se o trabalho detalhado de Zagal, Bindra e Yeager [33] com a ftalocianina tetrassulfonada de ferro, FeTsPc, um derivado solúvel em soluções aquosas e que se adsorve espontânea e irreversivelmente, a níveis de monocamadas, sobre superfícies de grafite pirolítico. Os eletrodos assim preparados apresentaram picos voltamétricos bem definidos e associados com processos redox envolvendo o centro metálico, bem como alta atividade eletrocatalítica para a redução de oxigênio em soluções aquosas alcalinas, via mecanismo direto e envolvendo espécies $\mathrm{Fe}(2+) \mathrm{TsPc}$ adsorvidas como eletroquimicamente ativas.

Apesar da baixa solubilidade das ftalocianinas e das dificuldades na preparação de derivados solúveis em água, Kobayashi e Nishiyama [32] investigaram a redução catalítica de oxigênio sobre eletrodos de carbono vítreo em soluções aquosas alcalinas contendo $\sim 3 \times 10^{-5} \mathrm{~mol}$ $\mathrm{L}^{-1}$ de ftalocianina tetracarboxilada de ferro, $\mathrm{FeTcPc}$ (Figura 1). Os experimentos indicaram que a reação na presença de $\mathrm{FeTcPc}$ em solução envolve 4 elétrons e processa-se segundo um mecanismo eletroquímico seguido de regeneração catalítica (EC) e envolvendo a formação de peróxido de hidrogênio como intermediário. Uma vez que processos redox superficiais não foram observados, espectros ultravioleta-visível e de dicroísmo circular magnético (MCD) foram registrados e indicaram as espécies $\mathrm{Fe}(2+) \mathrm{TcPc}$ de baixo spin como as responsáveis pela eletrocatálise. Todavia, com base no fato de que muitos estudos da redução de oxigênio catalisada por eletrodos modificados com ftalocianina de ferro, FePc, "adsorvida" apresentarem como espécies ativas os estados de spin intermediário e alto [35] e não o de baixo spin, Kobayashi e Nishiyama [32] concluíram que as deduções para os eletrocatalisadores adsorvidos muitas vezes não são necessariamente verdadeiras para os dissolvidos no eletrólito.

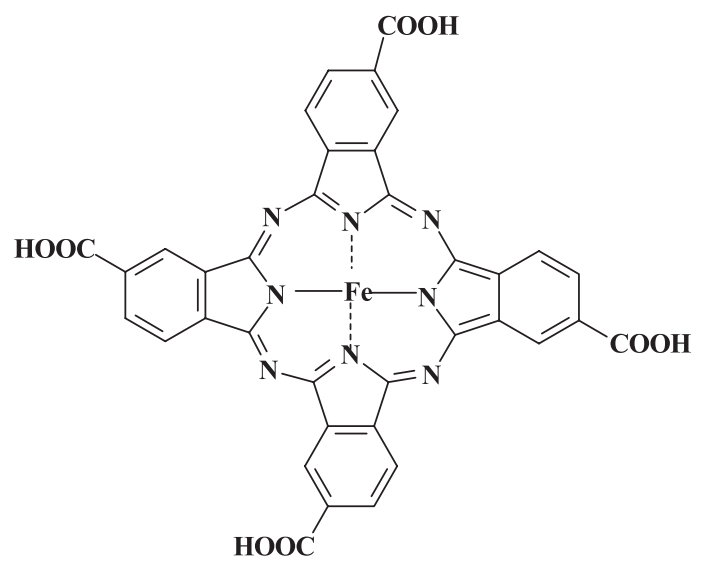

Figura 1. Estrutura da ftalocianina tetracarboxilada de ferro (FeTcPc). 
Por outro lado, experimentos realizados em nosso laboratório mostraram que, assim como o FeTsPc, o composto FeTcPc também se adsorve irreversivelmente, a níveis de monocamadas, sobre superfícies de grafite pirolítico. Esta observação pode ser atribuída à presença de diferentes grupos funcionais presentes na superfície do grafite pirolítico [36] que, muito provavelmente, em contraste com o carbono vítreo, conferem propriedades adsortivas adequadas para metaloftalocianinas e metaloporfirinas. Assim sendo, o presente trabalho foi elaborado com o propósito de se realizar um estudo sistemático das características eletroquímicas do derivado FeTcPc adsorvido na superfície do grafite pirolítico sem a sua presença na fase solução. Além disso, com base no estudo detalhado realizado com FeTsPc [33], comparar as propriedades eletrocatalíticas destes compostos adsorvidos para a redução de oxigênio em soluções aquosas alcalinas, uma vez ambos apresentam múltiplos picos voltamétricos na região de potenciais da redução de oxigênio.

\section{Procedimento Experimental}

Os experimentos eletroquímicos foram realizados em uma célula convencional de três compartimentos e utilizando-se um eletrodo de referência de calomelano saturado (ECS), adquirido da Analion Comércio e Indústria Ltda. (Ribeirão Preto - SP, Brasil), e um eletrodo auxiliar de platina $\left(\sim 2 \mathrm{~cm}^{2}\right.$ de área geométrica) da Heraeus Vectra do Brasil Ltda. (São Paulo - SP, Brasil).

O eletrodo de trabalho foi confeccionado na forma de eletrodo de disco rotatório, a partir de um cilindro de grafite pirolítico ordinário ("ordinary pyrolytic graphite"), da Union Carbide Co. (Cleveland - Ohio, USA), moldado em resina epóxi (Maxepoxi Ind. e Com. Ltda, São Paulo SP, Brasil), de modo a expor o plano basal ("basal plane") com uma área geométrica de $\sim 0,2 \mathrm{~cm}^{2}$. Para simplificação, este eletrodo será denominado eletrodo de grafite e denotado como GPB. Antes de cada experimento, o eletrodo GPB foi tratado com lixa de carbeto de silício de granulação 600 da 3M do Brasil (São Paulo - SP, Brasil) e posteriormente imerso, por cerca de cinco minutos, em água sonicada num sistema modelo
T7 da Thornton-Impec Eletrônica Ltda. (Vinhedo - SP, Brasil).

As medidas eletroquímicas foram realizadas com o auxílio de um bipotenciostato modelo AFRDE5 da Pine Instruments Co. (Grove City - PA, USA) acoplado a um registrador X-Y modelo 720M-30 da Allen Datagraph (New Hampshire, USA). As velocidades de rotação do eletrodo de trabalho foram controladas por um sistema também adquirido da Pine Instruments Co. e composto por um AFASR Rotator e um ASR Speed Control.

Antes do início de cada medida, a solução de trabalho era submetida à passagem de $\mathrm{N}_{2}$ ou $\mathrm{O}_{2}$ na célula eletroquímica, por cerca de 30 minutos. Estes gases tinham especificações de gases especiais analíticos 4.6 e 4.0, respectivamente, da White Martins S.A (São Luís - MA, Brasil).

Todas as soluções foram preparadas de acordo com a literatura [37] e utilizando-se reagentes de grau analítico e água destilada e purificada num sistema Milli-Q Academic adquirido da Millipore do Brasil S. A. (São Paulo - SP, Brasil). As seguintes soluções foram utilizadas: $\mathrm{NaOH}\left(0,1 \mathrm{~mol} \mathrm{~L}^{-1}\right.$ e 1,0 mol L-1); $\mathrm{NaOH} / \mathrm{Na}_{2} \mathrm{HPO}_{4}(\mathrm{pH} 12,0) ; \mathrm{Na}_{2} \mathrm{CO}_{3} / \mathrm{NaHCO}_{3}(\mathrm{pH}$ $10,0) ; \mathrm{NaOH} / \mathrm{H}_{3} \mathrm{BO}_{3} / \mathrm{KCl}(\mathrm{pH} 8,0) ; \mathrm{NaOH} /$ $\mathrm{KH}_{2} \mathrm{PO}_{4}(\mathrm{pH} 7,0) ; \mathrm{Na}_{2} \mathrm{HPO}_{4} / \mathrm{NaH}_{2} \mathrm{PO}_{4}(\mathrm{pH} 6,0)$; $\mathrm{NaOH} / \mathrm{KHC}_{8} \mathrm{H}_{4} \mathrm{O}_{4}(\mathrm{pH} 4,0) ; \mathrm{HCl} / \mathrm{KHC}_{8} \mathrm{H}_{4} \mathrm{O}_{4}(\mathrm{pH}$ $2,0) \mathrm{e}_{2} \mathrm{SO}_{4}\left(0,05 \mathrm{~mol} \mathrm{~L}^{-1}\right)$. Os valores dos $\mathrm{pH}$ das soluções foram medidos em um pHmetro modelo DMPH-1 da Digimed (São Paulo - SP, Brasil). O complexo FeTcPc foi gentilmente cedido pelo Prof. Dr. Daniel Scherson do Department of Chemistry da Case Western Reserve University (Cleveland - OH, USA).

$\mathrm{O}$ método adotado para as modificações do eletrodo GPB com FeTcPc foi o da adsorção irreversível [39]. Para isto, o eletrodo GPB era imerso em uma solução $1 \times 10^{-3} \mathrm{~mol} \mathrm{~L}^{-1}$ FeTcPc em $\mathrm{NaOH} 0,1 \mathrm{~mol} \mathrm{~L}^{-1}$ e deixado em contato por cerca de 10 minutos, sob uma rotação de $100 \mathrm{rpm}$. Posteriormente, o eletrodo GPB modificado com $\mathrm{FeTcPc}$ era lavado com água e imediatamente introduzido na célula eletroquímica. Este procedimento é suficiente e adequado para a produção de eletrodos de grafite modificados com monocamadas de metaloftalocininas adsorvidas irreversivelmente [16,27,39]. 


\section{Resultados e discussão}

Estudos voltamétricos das propriedades redox de eletrodos GPB modificados com FeTcPc

A Figura 2 mostra os voltamogramas cíclicos registrados sobre o eletrodo GPB antes e após modificação com FeTcPc em uma solução 0,1 mol L${ }^{1} \mathrm{NaOH}$ saturada com $\mathrm{N}_{2}$. O eletrodo GPB apresentou um voltamograma sem a presença de processos de transferência de carga, ou seja, com correntes de natureza puramente capacitivas [40]. Após modificação com FeTcPc, o voltamograma apresentou quatro picos voltamétricos bem definidos e localizados próximos a $-1,35 \mathrm{~V}$ (pico 1 ), $-0,45 \mathrm{~V}$ (pico 2), $-0,15 \mathrm{~V}$ (pico 3) e +0,40 V (pico 4). Estes picos apresentaram uma dependência diretamente proporcional entre as correntes de pico catódica $\left(I_{p c}\right)$ e anódica $\left(I_{p a}\right)$ com a velocidade de varredura do potencial $(v)$, bem como uma relação $\left|I_{p c} / I_{p a}\right| » 1$. Além disso, a separação entre os potenciais dos picos anódico e catódico $\left(\mathrm{DE}_{\mathrm{p}}\right)$ foi sempre muito pequena, com valores menores ou iguais a $20 \mathrm{mV}$, inclusive para a mais alta velocidade de varredura do potencial utilizada. Estas observações indicam que os picos voltamétricos apresentados pelo eletrodo GPB modificado com FeTcPc correspondem a processos de transferência de carga envolvendo somente espécies adsorvidas [27,28,31,33,40]. Os picos 2 e 3 têm sido reportados sobre eletrodos de carbono e grafite modificados com ftalocianina de ferro (FePc) $[27,29,30]$ e seus derivados tetrassulfonatado (FeTsPc) [33] e octametoxilado (FeMeOPc) [23], e associados a processos redox envolvendo o centro metálico, ou seja, $\mathrm{Fe}(1+) / \mathrm{Fe}(2+)$ e $\mathrm{Fe}(2+) / \mathrm{Fe}(3+)$, respectivamente. Além disso, a presença dos picos 1 e 4 foi inicialmente registrado sobre FeTsPc [31] e posteriormente sobre o composto tetrapiridinoporfirazina de ferro (FeTPyPz) [28], sendo atribuídos a processos redox envolvendo o anel do macrociclo $(\mathrm{Pc})$, ou seja, $\mathrm{Fe}(1+) \mathrm{Pc}(2-) /$ $\mathrm{Fe}(1+) \mathrm{Pc}(3-)$ e $\mathrm{Fe}(3+) \mathrm{Pc}(2-) / \mathrm{Fe}(3+) \mathrm{Pc}(1-)$, respectivamente.
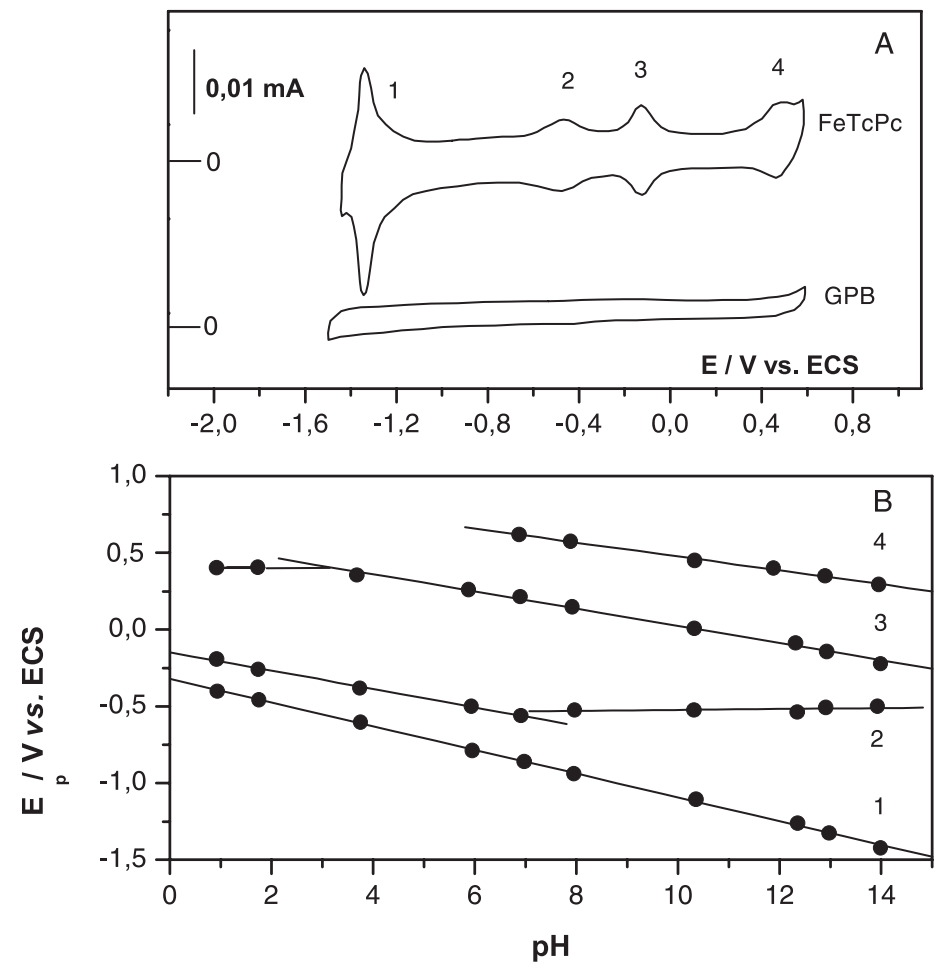

Figura 2. a) Voltamogramas cílicos registrados sobre o eletrodo GPB antes ( $v=100$ $\left.\mathrm{mV} \mathrm{s}^{-1}\right)$ e após modificação com FeTcPc $\left(\mathrm{v}=200 \mathrm{mV} \mathrm{s}^{-1}\right)$. Eletrólito: $0,1 \mathrm{~mol} \mathrm{~L}^{-1}$ $\mathrm{NaOH}$ saturado com $\mathrm{N}_{2}$, b) Dependência dos potenciais formais dos processos redox do FeTcPc adsorvido sobre GPB com o pH da solução. Temperatura ambiente. 
Para um dado pico voltamétrico, assumindo comportamento nernstiano ideal, a corrente de pico $I_{p}$ pode ser relacionada com a velocidade de varredura do potencial $v$ pela equação [40]:

$$
I_{p}=\left(\frac{n^{2} F^{2}}{4 R T}\right) v A \Gamma
$$

onde $\Gamma$ representa a concentração total de espécies adsorvidas sobre a superfície do eletrodo $\left.(\mathrm{mol} \mathrm{cm})^{2}\right)$, $A$ a área do eletrodo $\left(\mathrm{cm}^{2}\right)$ e os outros termos tem seus significados usuais. A concentração total de espécies adsorvidas está relacionada com a carga $Q$ sob o pico voltamétrico pela equação [40]:

$$
Q=n F A \Gamma
$$

Substituindo o produto $A$ da equação 2 na 1 e rearranjando o resultado em uma maneira mais simples, é possível obter a expressão:

$$
n=\frac{\widetilde{c}}{\mathrm{c}} \frac{\mathrm{e} T I_{p}}{v F Q} \frac{\grave{\dot{\dot{\zeta}}}}{\dot{\phi}}
$$

A partir desta equação e dos valores das cargas, calculadas com base nas áreas sob os picos voltamétricos da Figura 2, os valores obtidos para $n$ foram, em todos os casos, próximos a 1 , ou seja, cada processo redox, muito provavelmente, envolve 1 elétron por molécula adsorvida. Assim sendo e utilizando-se a equação 2 para o cálculo de espécies adsorvidas, foi encontrado um valor de aproximadamente $1,9 \times 10^{-10} \mathrm{~mol} \mathrm{\textrm {cm } ^ { - 2 }}$ para $\Gamma$. Este valor está bastante próximo dos valores $2,0 \times 10^{-10} \mathrm{~mol} \mathrm{~cm}^{-2}$ reportados por van der Putten e colaboradores [41] para FePc e $1,74 \times 10^{-10} \mathrm{~mol}$ $\mathrm{cm}^{-2}$ obtido por Zagal e outros [27] para diversas metaloftalocianinas, sob condições similares. Além disso, se admitirmos que a molécula FeTcPc possui uma área geométrica da ordem de 120 a $200 \AA^{2}$ por molécula $[27,33,41]$ e que cada molécula adsorve com o plano do anel macrociclo paralelo à superfície do eletrodo, o valor calculado para $\mathrm{G}$ corresponde ao valor aproximado de 1 monocamada de espécies adsorvidas.

A dependência dos potenciais formais
$E^{o}$ dos processos redox do composto FeTcPc adsorvido sobre GPB, mostrados na Figura 2, foi estudada em função do $\mathrm{pH}$ da solução e os resultados são apresentados na Figura 2. O pico 1 apresentou uma dependência da ordem de $60 \mathrm{mV}$ para cada variação unitária de $\mathrm{pH}$ enquanto o pico 4 apresentou uma inclinação de $50 \mathrm{mV} / \mathrm{pH}$ em soluções alcalinas e em soluções ácidas o processo redox não foi observado. A concordância destes valores com o valor teórico de $\sim 60 \mathrm{mV} / \mathrm{pH}$, calculado a partir da equação de Nernst, sugere que os processos envolvidos com os picos 1 e 4 e associados com a redução e oxidação do anel do macrociclo $\mathrm{Pc}$, respectivamente, são dependentes do $\mathrm{pH}$ do meio e envolvem um próton ou uma hidroxila para cada elétron da reação em questão. A seguir, as dependências dos picos 2 e 3 com o pH são abordados com maiores detalhes, pois são aqueles que envolvem o centro metálico e que estão diretamente associados com a atividade eletrocatalítica do eletrodo GPB/FeTcPc para a redução de oxigênio.

Em soluções de $\mathrm{pH}<7$, o pico 2 apresentou uma dependência de cerca de $60 \mathrm{mV} / \mathrm{pH}$ e, muito provavelmente, o processo redox esteja relacionado com a seguinte reação [28,31]:

$$
\begin{aligned}
& \left(\mathrm{H}_{2} \mathrm{O}\right)_{2} \mathrm{Fe}(2+) \mathrm{TcPc}(2-)+\mathrm{e}^{-}+\mathrm{H}^{+} \rightleftharpoons \\
& {\left[\left(\mathrm{H}_{2} \mathrm{O}\right)_{2} \mathrm{Fe}(1+) \mathrm{TcPc}(2-)\right]^{-} \mathrm{H}^{+}}
\end{aligned}
$$

Para soluções de $\mathrm{pH}^{3} 7$, o potencial tornase independente da variação do $\mathrm{pH}$ do meio, e a reação pode ser descrita como [28,31]:

$$
\begin{aligned}
& \left.\left(\mathrm{H}_{2} \mathrm{O}\right)_{2} \mathrm{Fe}(2+) \mathrm{TcPc}(2-)\right]+\mathrm{e}^{-} \\
& {\left[\left(\mathrm{H}_{2} \mathrm{O}\right)_{2} \mathrm{Fe}(1+) \operatorname{TcPc}(2-)\right]^{-}}
\end{aligned}
$$

O pico 3 em soluções de $\mathrm{pH}$ variando de 4 a 14 apresentou uma dependência de $60 \mathrm{mV} / \mathrm{pH}$ (Figura 3) e o processo redox associado, em meio alcalino, pode ser escrito na forma [28,31]:

$$
\begin{aligned}
& \left.(\mathrm{OH})\left(\mathrm{H}_{2} \mathrm{O}\right) \mathrm{Fe}(3+) \mathrm{TcPc}(2-)\right]+\mathrm{e}^{-}+\mathrm{H}_{2} \mathrm{O} \\
& \left.\left(\mathrm{H}_{2} \mathrm{O}\right)_{2} \mathrm{Fe}(2+) \mathrm{TcPc}(2-)\right]+\mathrm{OH}^{-}
\end{aligned}
$$


Para valores de $\mathrm{pH}$ menores ou igual a 3 não se observou uma variação do potencial, indicando que, nesse intervalo, o processo redox torna-se independente da concentração de prótons em solução [28,31]:

$$
\begin{aligned}
& \left.\left(\mathrm{H}_{2} \mathrm{O}\right)_{2} \mathrm{Fe}(3+) \operatorname{TcPc}(2-)\right]^{+}+\mathrm{e}^{-} \quad= \\
& \left(\mathrm{H}_{2} \mathrm{O}\right)_{2} \mathrm{Fe}(2+) \operatorname{TcPc}(2-)
\end{aligned}
$$

Estas reações assumem que o íon metálico possa estar hexacoordenado e, portanto, uma interação perpendicular do plano do macrociclo com a superfície do eletrodo. Note, contudo, que um conjunto similar de equilíbrios poderia ser escrito se um sítio de coordenação estivesse ligado à superfície do eletrodo (orientação paralela) por meio de um átomo de oxigênio. Neste caso, a interação do oxigênio axial com o eletrodo permaneceria intacta através de todos os estados de oxidação. Além disso, que ambos $\mathrm{Fe}(3+)$ e $\mathrm{Fe}(2+)$ podem estar hexacoordenados mas que $\mathrm{Fe}(1+)$ provavelmente esteja tetra ou pentacoordenado, dependendo da disponibilidade de ligantes "soft" [31].

Estudos voltamétricos das propriedades eletrocatalíticas de eletrodos GPB modificados com FeTcPc para a redução de oxigênio em soluções aquosas alcalinas

A Figura 3A apresenta os voltamogramas cíclicos da redução de oxigênio sobre o eletrodo GPB antes e após modificação com FeTcPc, em uma solução 0,1 mol $\mathrm{L}^{-1} \mathrm{NaOH}$. Os comportamentos observados são típicos de um processo irreversível e mostram que após a modificação houve um aumento acentuado na magnitude da corrente, bem como um deslocamento do potencial inicial de reação para valores bem mais positivos, cerca de $200 \mathrm{mV}$. Além disso, uma comparação destes resultados com os apresentados na Figura 2A indicam que a corrente de redução de $\mathrm{O}_{2}$ está diretamente associada com o processo redox $\mathrm{Fe}(3+) \mathrm{TcPc}(2-) /$ $\mathrm{Fe}(2+) \mathrm{TcPc}(2-)$, ou seja, que as espécies $\mathrm{Fe}(2+) \mathrm{TcPc}(2-)$ são as responsáveis pela atividade eletrocatalítica do complexo.

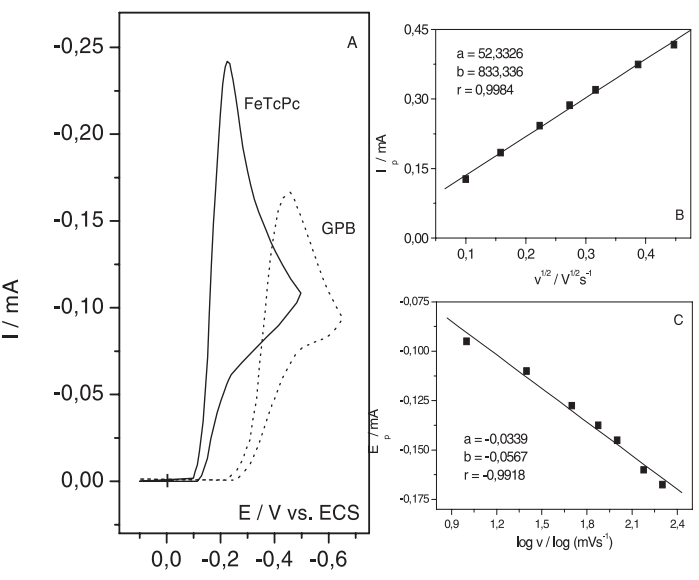

Figura 3. a) Voltamogramas cíclicos da redução de oxigênio sobre o eletrodo GPB antes e após modificação com FeTcPc ( $\mathrm{v}=50 \mathrm{mV} \mathrm{s}^{-1}$ ) e dependências a) de $I_{p} \operatorname{com} v$ e b) de $E_{p}$ com $\log v$, para a redução de oxigênio sobre eletrodos GPB modificados com FeTcPc. Eletrólito: $0,1 \mathrm{~mol} \mathrm{~L}^{-1} \mathrm{NaOH}$ saturado com $\mathrm{O}_{2}$. Temperatura ambiente.

Voltamogramas cíclicos da redução do oxigênio sobre FeTcPc em solução $0,1 \mathrm{~mol} \mathrm{~L}^{-1}$ $\mathrm{NaOH}$ também foram registrados a diferentes velocidades de varredura do potencial. Uma análise dos resultados mostrou uma dependência linear da corrente de pico I com a raiz quadrada da velocidade de varredura do potencial $v$ (Figura 3B), bem como do potencial de pico $\mathrm{E}_{\mathrm{p}} \operatorname{com} \mathrm{o} \log v$ (Figura 3C). Estas características são de um processo eletroquímico irreversível sob controle difusional e podem ser expressas pelas seguintes equações $[40,41]$ :

$$
\mathrm{I}_{\mathrm{P}}=2,99 \times 10^{5} n\left(\alpha n_{0}\right)^{1 / 2} \mathrm{~A} C_{0} D_{0}^{1 / 2} v^{1 / 2}
$$

$$
E_{P}=k+\left(\frac{0,03}{\alpha n_{0}}\right) \log v
$$

onde $C_{0}$ representa a concentração de $\mathrm{O}_{2}$ no seio da solução; $D_{0}$ o coeficiente de difusão do $\mathrm{O}_{2}$; $A$ a área do eletrodo; $n$ o número total de elétrons envolvidos na reação; $\alpha n_{0}$ um parâmetro que reflete a irreversibilidade da reação; $n_{0}$ o número de elétrons envolvidos na etapa determinante da velocidade da reação (e.d.v.) e $k$ uma constante. A combinação da Equação 9 com o coeficiente angular extraído da Figura 3C permitiu calcular o valor de $\alpha n_{0}$ como sendo de $\sim 0,535$. A partir deste 
valor e do coeficiente angular da Figura 3B substituídos na Equação $8 \mathrm{com} C_{0}=1,21 \times 10^{-6} \mathrm{~mol}$ $\mathrm{cm}^{-3}$ e $D_{0}=1,90 \times 10^{-5} \mathrm{~cm}^{2} \mathrm{~s}^{-1}[42,43]$, a $25^{\circ} \mathrm{Ce} A$ $=0,2 \mathrm{~cm}^{2}$, o valor calculado de $n$ foi de 3,7 indicando que a reação de redução de oxigênio catalisada pelo complexo FeTcPc em soluções aquosas alcalinas está associada com um processo envolvendo 4 elétrons. Neste sentido, experimentos de eletrodo de disco rotatório também foram realizados para a obtenção de maiores informações do mecanismo reacional envolvido, e os resultados são apresentados a seguir.

Estudos de eletrodo de disco rotatório para a redução de oxigênio sobre GPB modificado com $\mathrm{FeTcPc}$ em soluções aquosas alcalinas

A Figura 4A apresenta uma curva de polarização, registrada a uma velocidade de rotação do eletrodo de 1600 rpm para a redução de oxigênio em solução $0,1 \mathrm{~mol} \mathrm{~L}^{-1} \mathrm{NaOH}$ sobre GPB modificado com FeTcPc. Esta figura também apresenta, para efeito de comparação, a curva de polarização registrada nas mesmas condições sobre o eletrodo GPB não modificado. Após modificação da superfície GPB com FeTcPc, um aumento significativo nas magnitudes das correntes de redução de $\mathrm{O}_{2}$ e um deslocamento do potencial inicial de redução para valores bem mais positivos foram observados. O comportamento da curva de polarização pode ser descrita como composta de duas regiões: uma primeira região caracterizada por um acentuado aumento da corrente de redução a partir de $-0,08 \mathrm{~V}$ até atingir um valor limite da ordem de $-1,1 \mathrm{~mA}$ em potenciais próximos a $-0,5$ $\mathrm{V}$; e uma segunda região, compreendida entre os potenciais $-0,6$ e $-0,90 \mathrm{~V}$, onde se observa um decréscimo da corrente de redução para valores próximos a - $0,85 \mathrm{~mA}$. Assim como observado nos experimentos de voltametria cíclica, uma comparação da curva de polarização da redução de $\mathrm{O}_{2}$ sobre FeTcPc com as propriedades redox do complexo também sugere as espécies $\mathrm{Fe}(2+) \mathrm{TcPc}$ (pico 3 na Figura 2A) como as responsáveis pela eletrocatálise da reação.

As curvas de polarização apresentadas na Figura 4A demonstram que o eletrodo GPB modificado com FeTcPc apresenta atividade eletrocatalítica comparável à do eletrodo de platina (Pt), que catalisa a reação segundo um mecanismo 4 elétrons [1-4], bem como muito superior à do substrato de grafite, que promove a reação de acordo com um processo envolvendo 2 elétrons e formação de peróxido de hidrogênio como principal produto $[4,28]$. Além disso, uma comparação com as atividades eletrocatalíticas dos complexos FePc, FeTsPc e FeMeOPc [23,27] (Figura 4B) mostra que o FeTcPc apresenta correntes catalíticas máximas em potenciais mais positivos. Estas curvas de polarização apresentam uma região de correntes máximas ao invés de um verdadeiro patamar ("plateau"). O decréscimo na magnitude da corrente em potenciais mais negativos que $-0,50 \mathrm{~V}$ pode ser atribuída a uma mudança na natureza química ou estrutura do catalisador durante a redução do oxigênio [28,34,44], uma vez que nesta região o processo redox $\mathrm{Fe}(2+) \mathrm{TcPc} / \mathrm{Fe}(1+) \mathrm{TcPc}$ passa a ser operativo (pico 2 na Figura 2A). Esta mudança deve estar associada com um processo reversível pois as mesmas correntes máximas foram observadas quando o sentido da varredura do potencial foi revertida.
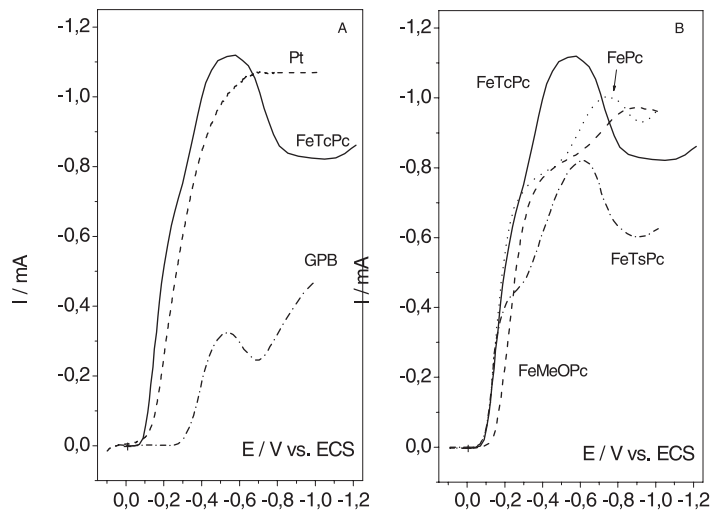

Figura 4. Curvas de polarização de estado estacionário para a redução de oxigênio sobre eletrodos a) Pt e GPB antes e após modificação com FeTcPc, e b) GPB modificados com FePc, FeTcPc, FeTsPc e FeMeOPc. Eletrólito: 0,1 $\mathrm{mol} \mathrm{L}^{-1} \mathrm{NaOH}$ saturado com $\mathrm{O}_{2}$. v $=10 \mathrm{mV} \mathrm{s}^{-1} \cdot f=1600 \mathrm{rpm}$. Temperatura ambiente.

Na Figura 5A são apresentadas as curvas de polarização da redução de $\mathrm{O}_{2}$ sobre o eletrodo GPB modificado com FeTcPc em solução $0,1 \mathrm{~mol}$ $\mathrm{L}^{-1} \mathrm{NaOH}$ para diferentes velocidades de rotação do eletrodo $f$. Para um dado potencial, se uma cinética de primeira ordem com relação ao oxigênio dissolvido estiver envolvida, a corrente 
observada $I$ está relacionada com i pela equação $[40,45,46]$ :

$$
\frac{1}{I}=\frac{1}{I_{K}}+\frac{1}{I_{D}}=\frac{1}{I_{K}}+\frac{1}{B f^{1 / 2}}
$$

onde $I_{K}$ representa a corrente cinética (ausência de qualquer efeito de transporte de massa) e $I_{D}$ a corrente difusional limite, dada pela equação:

$$
I_{D}=B f^{1 / 2}=\left(0,20 n F A D_{o}^{2 / 3} C_{o} v^{-1 / 6}\right) f^{1 / 2}
$$

onde $\mathrm{n}$ representa a viscosidade cinemática da solução e os outros termos possuem os mesmos significados mencionados anteriormente. Assim sendo, gráficos de $I^{-1}$ em função de $f^{-1 / 2}$ (gráficos de Koutecky-Levich) foram construídos para diferentes potenciais e são mostrados na Figura 5. Na região de potenciais entre $-0,25 \mathrm{~V}$ e $-0,40 \mathrm{~V}$ (Figura 5B), a linearidade e o paralelismo das retas observados sugerem que a Equação 10 é obedecida, ou seja, que a reação processa-se de acordo com uma cinética de primeira ordem em relação ao $\mathrm{O}_{2}$ e um valor constante no número de elétrons envolvidos na reação nesta região de potenciais. O valor médio do parâmetro $B$, extraído a partir dos coeficientes angulares, resultou ser da ordem de $3,12 \times 10^{-2} \mathrm{~mA}(\mathrm{rpm})^{-1 / 2}$. Este valor está em boa concordância com o valor teórico $2,94 \times 10^{-2} \mathrm{~mA}(\mathrm{rpm})^{-1 / 2}$, estimado para um processo obedecendo a Equação 11, $\operatorname{com} n=1,02 \times 10^{-}$ ${ }^{2} \mathrm{~cm}^{2} \mathrm{~s}^{-1}$ [15], $C_{0}=1,21 \times 10^{-6} \mathrm{~mol} \mathrm{~cm}{ }^{-3}, D_{0}=$ $1,90 \times 10^{-5} \mathrm{~cm}^{2} \mathrm{~s}^{-1}[41,42]$ a $25^{\circ} \mathrm{C}, A=0,2 \mathrm{~cm}^{2}$, e assumindo $n=4$. Portanto, assim como observado nos dados de voltametria cíclica, os resultados de disco rotatório também indicaram que a reação de redução de $\mathrm{O}_{2}$ sobre $\mathrm{FeTcPc}$ em soluções aquosas alcalinas processa-se de acordo com um mecanismo envolvendo 4 elétrons por molécula reagente (mecanismo direto):

$\mathrm{O}_{2}+2 \mathrm{H}_{2} \mathrm{O}+4 e^{-} \rightleftharpoons 4 \mathrm{OH}^{-}$

O paralelismo das retas não foi mantido para potenciais entre $-0,65 \mathrm{~V}$ e $-0,85 \mathrm{~V}$ (Figura $5 \mathrm{C})$. Os valores dos coeficientes angulares $(1 / B)$
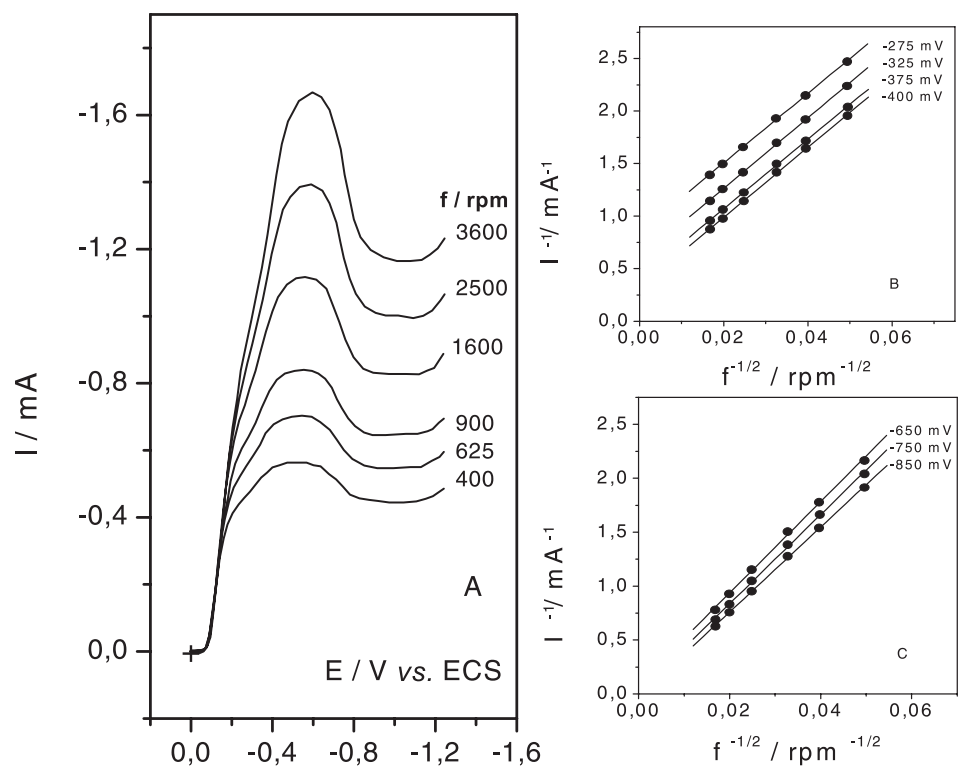

Figura 5. a) Curvas de polarização de estado estacionário registrados a diferentes velocidades de rotação do eletrodo para a redução de oxigênio sobre GPB modificado com FeTcPc. v= $10 \mathrm{mV} \mathrm{s}^{-1}$. b) e c) Gráficos de Koutecky-Levich para diferentes potenciais da redução de oxigênio sobre GPB modificado com FeTcPc. Dados extraídos da Figura 5a. Eletrólito: $0,1 \mathrm{~mol} \mathrm{~L}^{-1} \mathrm{NaOH}$ saturado com $\mathrm{O}_{2}$. Temperatura ambiente. 
aumentam à medida que o potencial torna-se mais negativo, indicando que o mecanismo da redução via 4 elétrons deixa de ser operativo e passa a ser gradualmente substituído por um mecanismo envolvendo 2 elétrons, com a geração de peróxido como produto da reação:

$\mathrm{O}_{2}+\mathrm{H}_{2} \mathrm{O}+2 \mathrm{e}^{-} \rightleftharpoons \mathrm{HO}_{2}^{-}+\mathrm{OH}^{-}$

Muito provavelmente, esta mudança no mecanismo está associada com o fato já mencionado de mudança na natureza química ou estrutura do catalisador durante a redução do oxigênio, com a interferência de espécies $\mathrm{Fe}(1+) \mathrm{TcPc}$ que não apresentam eficiência catalítica para a redução de oxigênio via 4-elétrons.

A elaboração de um mecanismo para a reação eletródica com múltiplas etapas, como é a reação de redução de oxigênio, bem como a identificação da etapa determinante da velocidade da reação, depende da análise dos diagramas de Tafel $[28,33,40]$ que, para uma dada velocidade de rotação do eletrodo, expressam a variação do potencial $E$ com o logaritmo da corrente $I$ corrigida do efeito de transporte de massa, ou seja, na forma $\log \left[I_{D} I / I_{D}-I\right]$. O diagrama de Tafel assim construído para a redução de $\mathrm{O}_{2}$ sobre FeTcPc em $0,1 \mathrm{~mol} \mathrm{~L}^{-1} \mathrm{NaOH}$ apresentou duas regiões lineares com coeficientes angulares da ordem de $-50 \mathrm{mV}$ década $^{-1}$ para a região de potenciais localizados no início da reação e de $-114 \mathrm{mV}$ década${ }^{1}$ para região de potenciais acima de $-0,20 \mathrm{~V}$. Estes valores são próximos dos registrados para os derivados FeTsPc, (-35 e -120 mV década ${ }^{-1}$ ) [33] e FeMeOPc (42 e -115 mV década $\left.{ }^{-1}\right)$ [23] sob condições similares e sugerem etapas química (Equação 14) e eletroquímica (Equação 17), respectivamente, como etapas determinantes da velocidade da reação [33]:

$$
\begin{aligned}
& \mathrm{Fe}(3+) \mathrm{TcPc}-\mathrm{OH}+\mathrm{e}^{-} \rightleftharpoons \mathrm{Fe}(2+) \mathrm{TcPc}+\mathrm{OH}^{-}(14) \\
& \mathrm{Fe}(2+) \mathrm{TcPc}+\mathrm{O}_{2} \rightleftharpoons \mathrm{Fe}(2+) \mathrm{TcPc}_{2} \mathrm{O}_{2} \\
& \mathrm{Fe}(2+) \mathrm{TcPc}_{-} \mathrm{O}_{2} \rightleftharpoons \mathrm{Fe}(3+) \mathrm{TcPc}_{2} \mathrm{O}_{2}^{-} \\
& \mathrm{Fe}(3+) \mathrm{TcPc}_{-} \mathrm{O}_{2}^{-}+\mathrm{e}^{-} \rightleftharpoons \text { Produtos }
\end{aligned}
$$$$
\text { Portanto, a reação de redução de oxigênio }
$$

em soluções aquosas alcalinas catalisada por eletrodos GPB modificados com FeTcPc processase segundo uma cinética de primeira ordem em relação ao oxigênio e segundo um mecanismo envolvendo 4 elétrons, com a formação de $\mathrm{OH}^{-}$ (Equação 12) como principal produto. A reação inicia-se em torno de -0,08 V vs. ECS e os dados de voltametria cíclica sugerem que o processo reversível envolvendo o centro metálico (Equação 14) ocorre na mesma região de potenciais onde a inclinação de Tafel mais baixa ( -50 mV década $\left.{ }^{-1}\right)$ é observada. Em potenciais mais negativos, a concentração superficial de espécies $\mathrm{Fe}(2+) \mathrm{TcPc}$ aumenta, assim como a corrente de redução de $\mathrm{O}_{2}$, e a velocidade da reação é controlada pelo processo de transferência de carga (Equação 17). Contudo, a conversão de todas as espécies $\mathrm{Fe}(3+) \mathrm{TcPc}$ em $\mathrm{Fe}(2+) \mathrm{TcPc}$ e suas interações com $\mathrm{O}_{2}$ produzem correntes limites em potencias próximos a $-0,45 \mathrm{~V}$. Em potenciais mais negativos que $-0,60 \mathrm{~V}$, o recobrimento do eletrodo com espécies $\mathrm{Fe}(2+) \mathrm{TcPc}$ torna-se zero e a corrente cai rapidamente, indicando que o mecanismo via formação de peróxido de hidrogênio (Equação 13) passa a ser operativo. Muito embora etapas posteriores à etapa determinante da velocidade da reação (Equação 17) não possam ser preditas, a formação de peróxido por meio da redução direta de oxigênio sobre áreas da superfície do GPB não recobertas com FeTcPc também não pode ser descartada [33].

Assim sendo, o presente mecanismo para a redução de oxigênio catalisada por FeTcPc adsorvido sobre eletrodos de grafite pirolítico difere do proposto por Kobayashi e Nishiyama [32] para eletrodos de carbono vítreo na presença de FeTcPc no eletrólito, onde a formação quantitativa de peróxido de hidrogênio foi proposta. De acordo com estes autores, a redução também inicia-se com a etapa eletroquímica dada pela Equação 14 e prossegue através de uma etapa de regeneração catalítica:

$$
\begin{aligned}
& \mathrm{Fe}(2+) \mathrm{TcPc}+1 / 2 \mathrm{O}_{2}+\mathrm{H}^{+}= \\
& \mathrm{Fe}(3+) \mathrm{TcPc}+1 / 2 \mathrm{H}_{2} \mathrm{O}_{2} \\
& \mathrm{Fe}(2+) \mathrm{TcPc}+1 / 2 \mathrm{H}_{2} \mathrm{O}_{2}+\mathrm{H}^{+} \rightleftharpoons \\
& \mathrm{Fe}(3+) \mathrm{TcPc}+\mathrm{H}_{2} \mathrm{O}
\end{aligned}
$$


Além disso, a possibilidade de uma sequência reacional com as seguintes etapas:

$$
\begin{aligned}
& \mathrm{Fe}(2+) \mathrm{TcPc}+\mathrm{H}_{2} \mathrm{O}_{2} \\
& \mathrm{Fe}(2+) \mathrm{TcPc}\left(\mathrm{H}_{2} \mathrm{O}_{2}\right) \\
& \mathrm{Fe}(2+) \mathrm{TcPc}\left(\mathrm{H}_{2} \mathrm{O}_{2}\right)+\mathrm{e}^{-} \\
& \mathrm{Fe}(3+) \mathrm{TcPc}+2 \mathrm{OH}^{-}
\end{aligned}
$$

ou

$\mathrm{Fe}(2+) \mathrm{TcPc}\left(\mathrm{H}_{2} \mathrm{O}_{2}\right)+\mathrm{Fe}(2+) \mathrm{TcPc} \rightleftharpoons$

$2 \mathrm{Fe}(3+) \mathrm{TcPc}+2 \mathrm{OH}^{-}$

também foi proposta.

Finalmente, experimentos da redução de oxigênio sobre FeTcPc em soluções aquosas ácidas também foram realizados, entretanto, demonstraram diminuições consideráveis nas correntes de redução para varreduras sucessivas do potencial, bem como um deslocamento do potencial inicial de redução para valores mais negativos, ou seja, em direção ao da curva de polarização do substrato GPB. Estas observações indicam que em soluções ácidas, FeTcPc apresenta acentuada instabilidade, com conseqüente dessorção e/ou degradação na superfície do eletrodo. Além disso, a insolubilidade de FeTcPc em soluções ácidas impediu qualquer tentativa da realização de experimentos com o composto presente no eletrólito de suporte.

\section{Agradecimentos}

Os autores agradecem ao CNPq (Processos 466.382/00-6 e 550.655/01-8) e à CAPES pelos auxílios financeiros e bolsas de estudos concedidos ao Grupo de Eletroquímica da UFMA.

J. A. P. Chaves; M. F. A. Araújo; J. de J. G. Varela Júnior; A. A. Tanaka. Eletrocatalysis of the oxygen reduction reaction on graphite electrodes modified with iron tetracarboxyphthalocyanine.

Abstract: This work presents a study of the electrochemical properties of pyrolytic graphite electrodes modified, at monolayer levels, with iron tetracarboxylated phthalocyanine, FeTcPc, in aqueous solutions. In alkaline media, the modified electrodes presented high electrocatalytic activity towards the oxygen reduction reaction, and comparable to that showed by platinum electrodes. The reaction proceeds according to a first order reaction with respect to dissolved oxygen, a mechanism involving 4 electrons and associated with the $\mathrm{Fe}(3+) \mathrm{TcPc} / \mathrm{Fe}(2+) \mathrm{TcPc}$ redox process.

Keywords: oxygen reduction, metallophthalocyanine, modified electrodes

\section{Referências bibliográficas}

[1] N. M. Markovic, P.N. Ross. Electrocatalysis at well-defined surfaces: kinetics of oxygen reduction and hydrogen oxidation/ evolution on Pt(hkl) electrodes. In: Wieckowski, A. Interfacial Electrochemistry - Theory, Experiments and Applications. New York: Marcel Dekker, 1999. 992p, 821-841.

[2] R. R. Adzic. Recent Advances in the Kinetics of Oxygen Reduction. In: LIPKOWSKI, J., ROSS, P. N. Electrocatalysis. New York, Wiley-VCH, 1998, pp.197-242.

[3] K. Kinoshita. Electrochemical Oxygen Technology. New York, Wiley-Interscience, 1992, 448p.

[4] M. R. Tarasevich, A. Sadkowski, E. Yeager. Oxygen electrochemistry. In: Conway, B. E., Bockris, J. O'M., Yeager, E., Khan, S. U. M., White, R. E.. Comprehensive Treatise in Electrochemistry. New York, Plenum Press, 1983, pp.301-398. [5] U. A. Paulus, A. Wokaun, G. G. Scherer, T. J. Schmidt, V. Stamenkovi, N. M. Markovic, P. N., Ross. Electrochimica Acta
47 (2002) 3787.

[6] S. C. Thomas, X. M. Ren, S. Gottesfeld, P. Zelenay. Electrochimica Acta 46 (2002) 3741.

[7] C. Lamy, A. Lima, V. Lerhun, F. Delime, C. Countanceau, J. M. LegeR. Journal of Power Sources 105 (2002) 283.

[8] P. Costamagna, S. Srinivasan. Journal of Power Sources 102 (2001) 242.

[9] T. J. P. Freire, E. R. Gonzalez. Journal of Electroanalytical Chemistry 503 (2001) 57.

[10] M. T. Giacomini, E. A. Ticianelli, J. McBreen, M.Salasubramanian. Journal of The Electrochemical Society 148 (2001) A323.

[11] A. J. McEvoy. Journal of Material Science 36 (2001) 1087. [12] A. A. Tanaka, R. Adzic, B. Nikolic. Jounal of Serbian Chemistry Society 64 (1999) 695.

[13] M. Calegaro, J. Perez, A. A. Tanaka, E. A. Ticianelli, E. Gonzalez. Denki Kagaku 64 (1996) 436.

[14] S. A. S. Machado, A. A. Tanaka, E. Gonzalez. 
Electrochimica Acta 39 (1994) 2591.

[15] J. Perez, A. A. Tanaka, E. Gonzalez, E. A. Ticianelli. Journal of The Electrochemical Society 141 (1994) 431.

[16] J. H. Zagal. Coordination Chemistry Reviews 119 (1992) 89.

[17] P. Vasudevan, H. Santosh, N. Mann, S. Tyagi. Transition Metal Chemistry 15 (1990) 81.

[18] M. R. Tarasevich, K. A. Radyushkina. Russian Chemical Reviews 49 (1980)718-730.

[19] N. Zheng, Y. Zeng, P. G. OsbornE, Y. Li, W. Chang, Wang. Journal of Applied Electrochemistry 32 (2002) 129.

[20] G. Ramirez, E. Trollund, M. Isaacs, F.Rmijo, J. Zagal, J. Costamagna, M. J. Aguirre. Electroanalysis 14 (2002) 540.

[21] S. L. P. Dias, Y. Gushikem, E. S. Ribeiro, E. V. Benvenutti. Journal of Electroanalytical Chemistry 523 (2002) 64.

[22] M. Ebadi, C. Lexiou, A. B. P. Lever. Canadian Journal of Chemistry 79 (2001) 992.

[23] R. P. Alves, C. D. C. Conceição, J. A. Chaves, A. A. Tanaka. Anais da Associação Brasileira de Química 49 (1)(2000) 46.

[24] E. H. Song, C. N. Shi, F. C. Anson. Langmuir 14 (1998) 4315. [25] A. Biloul, P. Gouerec, M. Savy, G.Carbeck, S. Besse, J. Riga. Journal of Applied Electrochemistry 26 (1996) 1139.

[26] N. Phougat, P. Vasudevan. Journal of Power Sources 69 (1997) 161

[27] J. H. Zagal, M. Paez, A. A. Tanaka, J. R. Santos Junior, C. Linkous. Journal of Electroanalytical Chemistry 339 (12)(1992) 13.

[28] A. A. Tanaka, C. Fierro, D. A. Scherson, E. Yeager. Material Chemistry and Physics 22 (3-4)(1989) 431.

[29] A. A. Tanaka, C. Fierro, D. Scherson, E. Yeager. Journal of Physical Chemistry 91 (14)(1987) 3799.

[30] A. van der Putten, A. Elzing, W. Visscher, E. Barendrecht. Journal of Electroanalytical Chemistry 214 (1-2)(1986) 523. [31] S. Zecevic, B. Simic-Glavaski, E. Yeager, A. B. P. Lever, P. C. Minor. Journal of Electroanalytical Chemistry 196 (2)(1985) 339.
[32] N. Kobayashi, Y. Nishiyama. Journal of Physical Chemistry 89(1985) 1167.

[33] J. H. Zagal, P. Bindra, Yeager, E. Journal of The Electrochemical Society 127 (7)(1980) 1506.

[34] J. P. Collman, P. DENISEVICH, Y. Konai, M. Marrocco, C. Koval, F. C. Anson. Journal of The American Chemical Society 102 (1980) 6027.

[35] A. J. Appleby, J. Fleisch, M. Savy. .Journal of Catalysis 44(2)(1976) 281.

[36] K. Kinoshita. Carbon: Electrochemical and Physicochemical Properties. New York. Wiley-Interscience, 1987. 533p.

[37] D. D. Perrin, B. Dempsey. Buffers for $\mathrm{pH}$ and Metal Ion Control. New York: Chapman and Hall, 1974. 171p.

[38] K. D. Snell, A. G. Keenan. Chemical Society Reviews 8 (1979) 259

[39] M. S. R. Nahuz, A. P. Reis, M. F. A. Araújo, A. A. Tanaka. Influências das Orientações Basal e Edge e do Pré-Tratamento Superficial nas Propriedades Eletroquímicas de Eletrodos de Grafite Pirolítico Modificados com Ftalocianina de Cobalto", Cadernos de Pesquisa da Universidade Federal do Maranhão, v.11 (1), p.65-74, 2000.

[40] A. J. Bard, L. R. Faulkner. Electrochemical methods. New York: John Wiley \& Sons, $1980.718 \mathrm{p}$

[41] R. S. Nicholson, I. Shain. Analytical Chemistry 36 (4)(1964) 706.

[42] K. E. Gubbins, R. D. Walker. Journal of The Electrochemical Society 112 (5)(1965) 469.

[43] R. E. Davis, G. L. Horvath, C. W. Tobias. Electrochimica Acta 12 (3)(1967) 287.

[44] J. J. Zhang, F. C. Anson. Journal of Electroanalytical Chemistry 341 (1-2)(1992) 323.

[45] J.Koutecky, B. G. Levich. Zhurnal Fizicheskoi Khimii 32 (7)(1958) 1565.

[46] B. G. Levich. Physicochemical Hydrodynamics. Englewood Cliffs - NJ: Prentice Hill, 1962, p.345. 